\title{
ACTIVE CONTROL OF SMART TENSEGRITY STRUCTURES
}

\author{
A. AL SABOUNI-ZAWADZKA ${ }^{1}$
}

\begin{abstract}
The topic of smart structures, their active control and implementation, is relatively new. Therefore, different approaches to the problem can be met. The present paper discusses variable aspects of the active control of structures. It explains the idea of smart systems, introduces different terms used in smart technique and defines the structural smartness. The author indicates differences between actively controlled structures and structural health monitoring systems and shows an example of an actively controlled smart footbridge.

The analyses presented in the study concern tensegrity structures, which are prone to the structural control through self-stress state adjustment. The paper introduces examples of structural control performed on tensegrity modules and plates. An influence of several self-stress states on displacements is analyzed and a study concerning damage due to member loss is presented.
\end{abstract}

Keywords: smart structure, active control, tensegrity, member loss, deployable footbridge

\section{INTRODUCTION}

Many engineering structures, such as buildings or bridges, are subject to various actions, among which the most dangerous ones are accidental loads. It may be a challenge for an engineer to design a structure, that would react to such accidental loading by modifying its own properties. It is possible with the use of smart technologies. Such intelligent technologies apply to both materials and structures. However, considering the immense costs of implementing smart materials into structural elements, the author focuses on structural smartness rather than the material one.

The present paper discusses variable aspects of an active control of structures. It explains the idea of smart systems, introduces different terms used in smart technique and defines the structural smartness (Gilewski, Al Sabouni-Zawadzka [1]; Cazzulani et al. [2]; Strong, Jensen [3]). The author indicates differences between actively controlled structures and structural health monitoring systems and presents an example of an actively controlled smart footbridge.

Buildings or bridges can be regarded as smart or intelligent due to the advanced structural health monitoring (SHM) systems, which are very often installed on the existing and newly-built structures. However, the author suggests a slightly different

1 Warsaw University of Technology, Faculty of Civil Engineering, Al. Armii Ludowej 16, 00-637 Warsaw, Poland, e-mail: a.sabouni@il.pw.edu.pl 
approach to the topic of smart structures. According to the author, "smartness" should regard a structure itself, not the advanced technologies, with which buildings or bridges are equipped. Intelligent systems, understood in such way, may have various applications: vibration damping, reduction of displacements, acoustic isolation, damage localization and self-repair, stress reduction, etc.

There are several research units which explore or explored the topic of smart structures for civil engineering applications. Some of their research projects are presented below.

A group of scientists from Ecole Polytechnique Fédérale de Lausanne has conducted different research on smart tensegrity structures (Adam, Smith [4]; Korkmaz et al. [5]; Rhode-Barbarigos et al. [6]). One of their projects concerned a full-scale model of a smart modular tensegrity structure, equipped with three displacement sensors and ten actuators. The structure was controlled by the active modification of self-stress state between struts and cables. The primary objective of the control was to maintain a constant slope of the upper surface of the structure. The control was performed by modifying lengths of the struts. When it came to local damage, the actively controlled smart system compensated the broken element, satisfying the serviceability criteria.

Another research project carried out by the scientists from Lausanne concerned a smart tensegrity footbridge. The structure was equipped with health monitoring system and a set of actuators installed on the selected cables. It was controlled through the active modification of prestressing forces, which was achieved by changing lengths of the cables. The main aim of the control was to ensure a constant level of stress values in structural elements. After local damage of the footbridge, realized by removing one of the cables, the smart system was able to compensate the broken element and satisfy the ultimate limit state criteria.

A research unit from the Smart Structures Technology Laboratory at the University of Illinois at Urbana-Champaign (Chang, Spencer [7]), on the other hand, investigated a scale model of the smart two-storey building. The steel structure was equipped with six low-friction pendulous bearings and three low-force hydraulic actuators. The aim of the control was to limit the base displacements, reducing in the same time the floor accelerations. The experiment was carried out in order to compare passive and active base isolation systems.

Another project - research on a smart carbon fibre structure - was conducted at Politecnico di Milano (Cazzulani et al. [8]). The simple structure, composed of a thin cantilever, was equipped with fourteen longitudinal FBG sensors and three PZT actuators. The control consisted in the stress and vibration reduction through the active damping and dissipation of the mechanical energy of the structure.

Scientist from the National Taiwan University, contrariwise, researched a smart sensing system for a continuous structural health monitoring ( $\mathrm{Lu}$ et al. [9]). The system, consisting of sensors, actuators and two servers, was installed on the new Civil Engineering research building of the National Taiwan University constructed on July 2008. While sensors were responsible for recording the response of the structure to the 
time-varying loads, the aim of the actuators was to reduce the structural response and avoid global damage of the building. Two servers took care of archiving the structural responses and data processing. In the eight-storey precast reinforced concrete building, two types of monitoring were applied. Whereas the first system controlled the seismic response of the structure during earthquake excitation, the second monitored continuously ambient vibrations of the building.

Analysing the presented literature, one may notice that there are various approaches to the concept of smart structures. According to the author's opinion, one of the most interesting possibilities of structural control lies in control of tensegrity structures through self-stress state adjustment (Skelton, Oliveira [10]; Motro [11]; Juang et al. [12]). Results of the performed analyses indicate that the structural displacements might be significantly reduced by adjusting the initial self-stress state in structural elements. The paper introduces examples of structural control performed on tensegrity modules and plates. An influence of several self-stress states on displacements is analyzed and a study concerning damage due to member loss is presented (Al Sabouni-Zawadzka, Gilewski [13]).

\section{WHAT IS A SMART STRUCTURE?}

The definition of smart structures has been a disputable issue for as long as the term exists. One of the problems that have to be considered in order to define a smart structure is the issue of a proper understanding of the word "smart". According to the dictionary, its original significance was "stinging, sharp". The present meaning of the word "smart" - "clever, intelligent" - has taken over from its original definition the element of quick energetic movement and sharp thought. This original meaning characterizes perfectly the idea of smart structures - structures that are capable of acting in a quick way and making corrections that resemble human decisions, particularly in response to variable conditions. The present significance, "intelligent", is also applied to the structures, but it is not fully adequate. Intelligence is a psychological term, a human feature and should be reserved for humans. Although the classification distinguishes a group of very smart structures, calling them intelligent, it does not mean that these structures possess intelligence. The only attribute, which makes them resemble humans, is the ability to learn.

To summarize, the author suggests:

- accepting the term "smart structure" as the only technical term used in relations to the structures,

- understanding structural "smartness" as an ability to evaluate the current situation and find the proper response,

- completing structural "smartness" with the original significance of the word, thus adding the feature of quick action to the definition of smart systems.

Another issue that should be considered is how a given group of people understands the term. A smart building, for example, can be understood in many different ways. 
For an occupant of the building it will signify a high-tech equipment, such as electronics controlling ambient environment, air conditioning systems, lighting and alarm installations. For an engineer designing the structure it will indicate that the building is equipped with a structural health monitoring and a damping system. For eco-conscious users it will mean applying smart ecological solutions such as energy saving systems and eco-friendly materials. The author, however, will focus on the meaning related to the structure itself and its behaviour, not its equipment or the materials used.

There are three terms connected with the topic of smart structures (Figure 1).

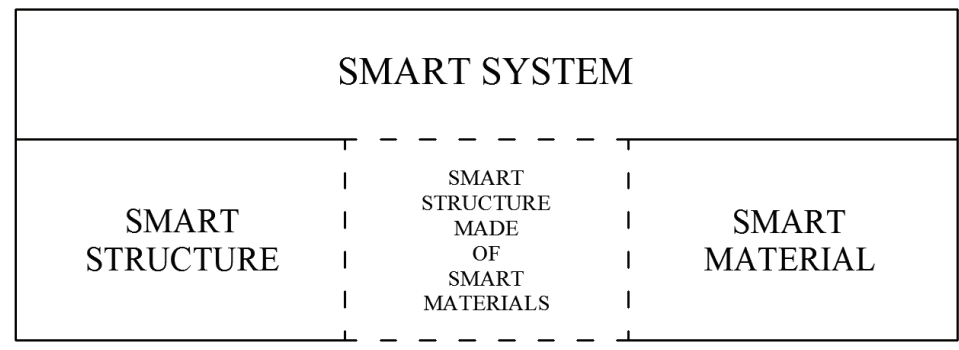

Fig. 1. Schematic diagram showing a smart concept

Smart structures are the structures with the ability to sense and respond adaptively to changes in their environment. This feature distinguishes them from the conventional ones. Whereas the main purpose of the traditional structures is to provide strength and carry loads acting on them, the smart ones adapt in a pre-designed manner to a functional need, modifying their shape, stiffness or damping characteristics in order to minimize deflection and possible damage.

Smart materials are the materials which are able to convert one form of energy (mechanical, magnetic, electrical, etc.) into another in a reversible and repeatable process. They are capable of sensing changes in the environmental conditions, responding to them in a predetermined manner, in an appropriate time and returning to their original shape as soon as the stimulus is removed. Smart materials are often used in actuation systems of smart structures, stimulating them to adapt to the variable conditions.

Smart systems are the systems composed of a smart material, a smart structure and an expert data processing. Smart systems ensure that during normal conditions, the structure carries all the loads without any help of smart components and on the other hand, it uses specific actuation systems to tackle abnormal load cases.

Another important concept related to the topic of smart structures is learning control (Adam, Smith [4]). The point of this phenomenon, also known as a case-based reasoning, is that the structure has a base, in which variable possible cases and control commands are stored. When the structure is subjected to a load, the suitable case is applied and the structure receives a certain command. As time goes by and different load cases arise, the structure adapts past control demands to the new loads. This ensures that 
the structure improves progressively its behaviour, reacting to the abnormal loads each time more rapidly.

The structures with the ability of learning are often called intelligent or very smart. They not only respond in a pre-designed manner, but also have the capability of adapting to the new conditions.

\section{ACtiVE CONTROL OF SYSTEMS VS. STRUCTURAL HEALTH MONITORING}

It can be found in the literature (Wilde [14]; Phares [15]; Eckhoff et al. [16]) that the terms: "active control" and "structural health monitoring" are used interchangeably. However, the author suggests distinguishing between the two of them.

The main idea of an actively controlled smart structure (Akhras [17]) is the integration of sensors, actuators and control mechanisms into one, fully functional and coherent system that becomes an integral part of the structure. Such system is able to sense changes in the environmental conditions and react to the stimulus in a predicted way, in real or close to real time. Its important feature is the use of feedback system in order to improve sensing and reaction processes.

Each smart system consists of three key elements: sensors, actuators and a control unit (Figure 2a).

a)

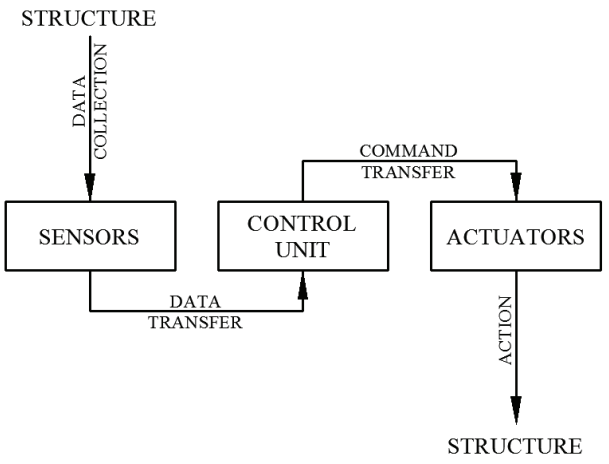

b)

STRUCTURE

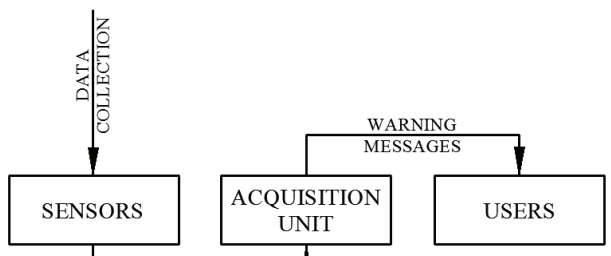

Fig. 2. Components of: a) actively controlled smart systems; b) structural health monitoring systems.

Sensors are the elements responsible for structural health monitoring. They detect changes in the environment, record the structural response (stress, strain, etc.) and generate appropriate signals which are then sent to the control unit.

Control unit is the element responsible for data analysis. The control centre gathers all the information received from sensing devices, processes them and, basing on the given algorithm, reaches the conclusion about further action. If the specific response is required, the control unit sends a signal to the appropriate actuator. 
Actuators are the elements responsible for reduction of the structural response. They change properties of the structure by applying a force that was computed by the control unit. This makes it possible to reduce the structural damage and avoid the catastrophic global collapse.

These elementary components work together using signal transferring devices which transmit: first collected data and then produced control commands between separate parts of the system.

A typical structural health monitoring (SHM) system, on the other hand, consists of: sensors, that are embedded in the structural elements or attached to their surface, cables and a data acquisition unit (Figure 2b). The aim of SHM is to detect structural damage, identify its type and location and determine the current state of the structure. The system can work continuously, performing measurements at regular intervals, or it might be used as a temporary monitoring. After each measurement, all data are sent to the data acquisition centre, where they are processed and analysed. In case of any anomaly or exceeding the maximum set values, the computer sends warning messages that inform the earlier defined units about potential danger.

The SHM system is the key element of all smart systems, but it does not mean that the structure equipped with such monitoring system is actively controlled. What is missing here is a feedback system. In each smart system, the data gathered by sensors is sent to the control unit, where it is analysed and the proper decision is made. If any parameter is exceeded, the control unit sends information to actuators, which are responsible for the reduction of structural response. In the SHM system, the described process ends on the level of data analysis. Of course, as a result of such analysis, the proper decisions concerning structural control are made, but this action involves human engagement, it does not function automatically.

To summarize, one has to combine three elements to obtain an actively controlled smart structure: structural health monitoring, a control unit and actuators. Only then will it be a complete smart system with the ability of health monitoring, damage detection, self-diagnostics and self-repair.

\section{General Characteristics of tensegrity Structures}

Tensegrities are cable-strut structures with a special node configuration, which ensures occurrence of an infinitesimal mechanism balanced with a self-stress state (Skelton, Oliveira [10]; Motro [11]; Gilewski, Kasprzak [18]). Tensegrities consist of struts (marked on the drawings with thicker lines), which can be compressed, and cables which are solely tensioned.

Taking into account mechanics of tensegrity structures, they can be considered trusses and described using the following parameters (unsupported truss):

$\mathbf{q}$ - vector of nodal displacements - length $M$, 
$\Delta-$ vector of strains in truss elements - length $N$,

$\mathbf{S}$ - vector of internal forces in truss elements - length $N$,

$$
\mathbf{E}=\left[\begin{array}{cccc}
\frac{E_{1} A_{1}}{L_{1}} & 0 & \ldots & 0 \\
0 & \frac{E_{2} A_{2}}{L_{2}} & \ldots & 0 \\
\ldots & \ldots & \ldots & \ldots \\
0 & 0 & \ldots & \frac{E_{N} A_{N}}{L_{N}}
\end{array}\right] \text {-stiffness matrix, }
$$

$\mathbf{P}$ - vector of nodal loads - length $\boldsymbol{M}$.

Relations between these parameters can be described as follows:

$$
\begin{gathered}
\boldsymbol{\Delta}=\mathbf{B}_{\mathbf{q}}, \\
\mathbf{S}=\mathbf{E} \boldsymbol{\Delta}, \\
\mathbf{B}^{T} \mathbf{S}=\mathbf{P},
\end{gathered}
$$

where: B - matrix of strains in truss elements, which allows to express strains using nodal displacements.

After inserting Eq. (4.1) and Eq. (4.2) into Eq. (4.3), a system of displacement-based equations is obtained:

$$
\mathbf{K}_{L} \mathbf{q}=\mathbf{P},
$$

where: $\mathbf{K}_{L} \mathbf{B}^{T} \mathbf{E B}$ - linear stiffness matrix.

In order to consider nonlinearity of equations of equilibrium and influence of selfstress on structural properties, geometric stiffness matrix $\mathbf{K}_{G}$ should be introduced. Then, the system of displacement-based equations will look as f ollows:

$$
\mathbf{K q}=\mathbf{P} \text {, }
$$

where: $\mathbf{K}=\mathbf{K}_{L}+\mathbf{K}_{G}$.

A system of stress-based equations, on the other hand, can be obtained by symmetrisation of Eq. (4.3):

$$
\mathbf{D S}=\mathbf{B P},
$$

where: $\mathbf{D}=\mathbf{B B}^{T}$. 
Matrix $\mathbf{B}$ can be determined using the finite element method formulation, according to the following algorithm:

1. Determination of nodal coordinates.

2. Determination of lengths and direction cosines of elements.

3. Determination of generalized Boolean matrices for elements $\mathbf{C}_{e}(e=1, \ldots, N)$, dimensions $M \times 2$ - allocation and transformation.

4. Determination of strains in elements: $\boldsymbol{\Delta}_{e}=\mathbf{b}_{e} \mathbf{q} ; \quad \mathbf{b}_{e}=\mathbf{B}_{e} \mathbf{C}_{e} ; \quad \mathbf{B}_{e}=\left[\begin{array}{ll}-1 & 1\end{array}\right]$.

5. Determination of matrix $\mathbf{B}$ from the row matrices $\mathbf{b}_{e} ; \mathbf{B}=\left[\begin{array}{c}\mathbf{b}_{1} \\ \mathbf{b}_{2} \\ \ldots \\ \mathbf{b}_{N}\end{array}\right]$.

Linear stiffness matrix $\mathbf{K}_{L}$ can be determined from the expression $\mathbf{K}_{L}=\mathbf{B}^{T} \mathbf{E B}$ or using the aggregation of linear stiffness matrices of single elements $\mathbf{K}_{L e}$, transformed from the local coordinate system into the global one by means of generalized Boolean matrices:

$$
\begin{gathered}
\mathbf{K}_{L e}=\frac{E_{e} A_{e}}{L_{e}}\left[\begin{array}{cc}
1 & -1 \\
-1 & 1
\end{array}\right], \quad \mathbf{q}_{L e}=\left\{u_{1}, u_{2}\right\}, \\
\mathbf{K}_{L}=\sum_{e=1}^{N} \mathbf{C}_{e}^{T} \mathbf{K}_{L e} \mathbf{C}_{e} .
\end{gathered}
$$

Global matrix $\mathbf{K}_{G}$ can be determined likewise $\mathbf{K}_{L}$, using generalised Boolean matrices $\overline{\mathbf{C}}_{e}$ (dimensions $M \times 6$ ) and a local element matrix $\mathbf{K}_{G e}$ :

$$
\mathbf{K}_{G}=\sum_{e=1}^{N} \overline{\mathbf{C}}_{e}^{T} \mathbf{K}_{G e} \overline{\mathbf{C}}_{e}
$$


In case of a supported truss, vector of nodal displacements $\tilde{\mathbf{q}}$ has smaller length $\tilde{N}$. Equations (Eq. 4.1-4.6) of linear mechanics of trusses change into the following expressions:

$$
\begin{gathered}
\boldsymbol{\Delta}=\widetilde{\mathbf{B}} \widetilde{\mathbf{q}}, \\
\mathbf{S}=\mathbf{E} \boldsymbol{\Delta}, \\
\widetilde{\mathbf{B}}^{T} \mathbf{S}=\widetilde{\mathbf{P}}, \\
\widetilde{\mathbf{K}} \widetilde{\mathbf{q}}=\widetilde{\mathbf{P}}, \\
\widetilde{\mathbf{K}} \widetilde{\mathbf{q}}=\widetilde{\mathbf{P}}, \\
\widetilde{\mathbf{D}} \mathbf{S}=\widetilde{\mathbf{B}} \widetilde{\mathbf{P}},
\end{gathered}
$$

where: $\widetilde{\mathbf{K}}_{L}=\widetilde{\mathbf{B}}^{T} \mathbf{E} \widetilde{\mathbf{B}}, \widetilde{\mathbf{K}}=\widetilde{\mathbf{K}}_{L}+\widetilde{\mathbf{K}}_{G}, \widetilde{\mathbf{D}}=\widetilde{\mathbf{B}} \widetilde{\mathbf{B}}^{T}$

Analysis of truss matrices allows to check whether or not the examined structure has tensegrity features. By analysing eigenvalues and eigenvectors of stiffness matrices: $\widetilde{\mathbf{K}}_{L}, \widetilde{\mathbf{K}}$ and matrices $\widetilde{\mathbf{D}}$, and by analysing strain matrices $\widetilde{\mathbf{B}}$, infinitesimal mechanisms and self-stress states might be identified.

As far as the linear stiffness matrix is concerned, the following eigenvalue problem for the supported truss is considered:

$$
\left(\widetilde{\mathbf{K}}_{L}-\lambda \mathbf{I}\right) \widetilde{\mathbf{q}}=\mathbf{0} .
$$

Eigenvalues describe energy states of the structure, while eigenvectors refer to deformation forms. In the correctly supported tensegrity structure, one (or more) of the eigenvalues equals zero and has a corresponding eigenvector which realizes the infinitesimal mechanism.

Taking matrix $\widetilde{\mathbf{B}}$ into account, in the correctly supported tensegrity structure its determinant equals zero, which is a result of the existing self-stress state. Values of internal forces related to self-stress can be determined analogically to eigenvectors - by assuming the value of one force and determining other forces depending on the assumed value.

After determining self-stress state, a geometric stiffness matrix $\widetilde{\mathbf{K}}_{G}$ can be built. The following eigenvalue problem is considered:

$$
\left(\widetilde{\mathbf{K}}_{L}+\widetilde{\mathbf{K}}_{G}-\lambda \mathbf{I}\right) \widetilde{\mathbf{q}}=\mathbf{0} .
$$


In the correctly supported tensegrity structure with a non-zero self-stress state, all the eigenvalues of the stiffness matrix $\widetilde{\mathbf{K}}=\widetilde{\mathbf{K}}_{L}+\widetilde{\mathbf{K}}_{G}$ are positive, which means that the matrix is invertible. Self-stress state removes singularity of the matrix and thus removes the infinitesimal mechanism.

\section{Active CONTROL OF TENSEGRITY STRUCTURES WITH GEOMETRICALLY NON-LINEAR} ANALYSIS

There are various regular and irregular tensegrity structures, including a series of typical modules, which can be used as a basis to build more complex systems.

Example of a tensegrity module - a 3-strut Simplex - is presented in Figure 3a. When the applied load acts in a direction of the infinitesimal mechanisms motion, it is possible to control stiffness of the module using relatively small values of prestressing forces. This feature of tensegrity structures is presented on the diagram (Figure 3b), showing how the value of prestressing force influences characteristic displacement of the given node. The analysis was performed in two steps: first the second-order theory was used and then the calculations were repeated using the third-order theory. The second-order theory can be used to identify infinitesimal mechanisms and to determine values of internal forces in self-stress states. The geometrically non-linear analysis (the third-order theory), on the other hand, should be used in engineering applications, when the values of displacements, internal forces and stresses are taken into account.

Analysis of the tensegrity module was performed using the following data: Young modulus of steel: $210 \mathrm{GPa}$, cross-sectional area of struts: $7,26 \mathrm{~cm}^{2}$, cross-sectional area of cables: 2,01 cm2, force P: $20 \mathrm{kN}$ (Figure 4).

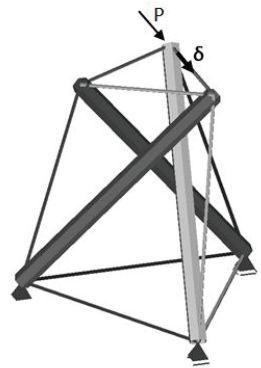

a)

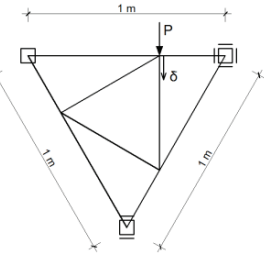

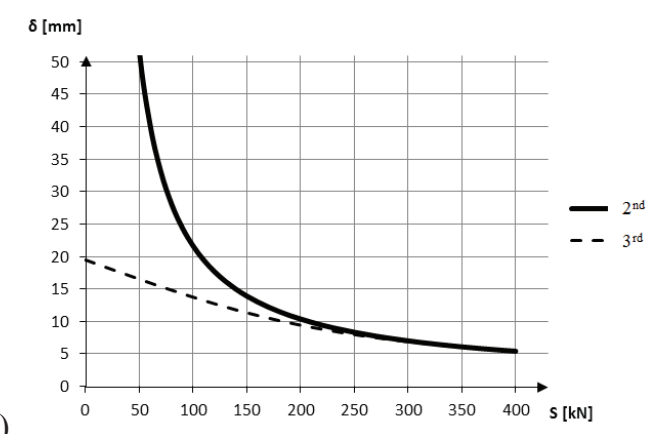

b)

Fig. 3. Single tensegrity module: a) geometry; b) influence of self-stress on the characteristic displacement.

The single tensegrity module has one infinitesimal mechanism and a corresponding self-stress state, which stiffens it. In case of any member loss, the module is subject to 
damage and collapses. Another behaviour characterizes the more complex structures, consisting of the combined tensegrity modules.

Figure 4a shows a plate structure, built of 14 reversed Simplex modules. The modules were located and combined in such a way that their lower nodes met. The plate was supported in four points on the upper surface and loaded with 13 point loads applied to the upper nodes of the plate, acting downwards.

The structure, similarly to the single tensegrity module, has one infinitesimal mechanism which allows to control the plate using relatively small values of prestressing forces (Figure $4 \mathrm{~b}$ ). Unlike Simplex, however, the tensegrity plate is not subject to damage in case of member loss and, what is even more important, has the ability of self-repair using self-stress.

The analysis was performed using the third-order theory and the same data as in the analysis of the single module. Two values of external forces P were considered: $10 \mathrm{kN}$ and $20 \mathrm{kN}$.

a)

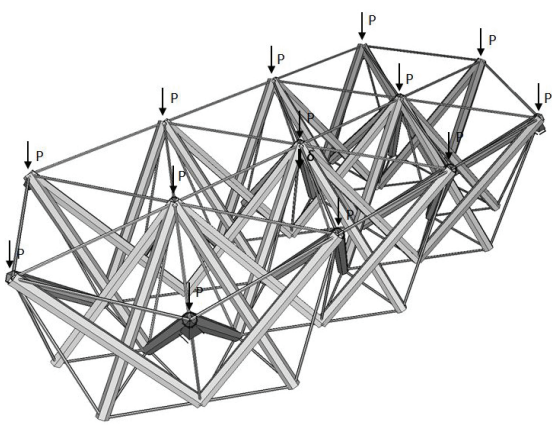

b)

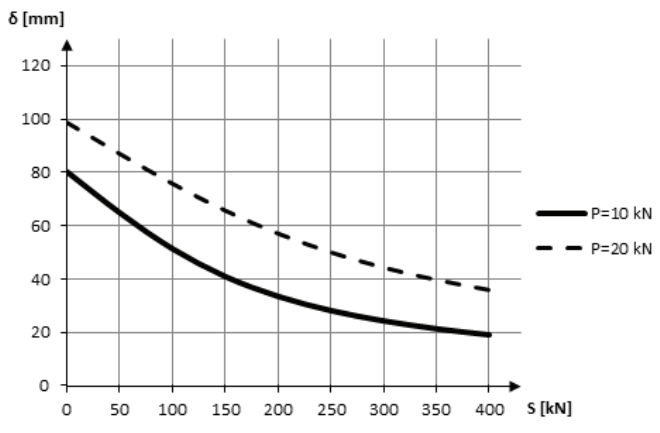

Fig. 4. Tensegrity plate: a) geometry; b) influence of self-stress on the characteristic displacement.

Figure 5a and 5b show how the values of internal forces in the selected elements ( 1 - an oblique cable, 2 - a strut) of the central module of the tensegrity plate change, depending on the value of external load. It can be observed that the increase of the values $\mathrm{S}_{\mathrm{f} 1}$ and $\mathrm{S}_{\mathrm{f} 2}$ diminishes when the value of prestressing forces grows, which means that the structure stiffens itself. The exact values of these forces are given in the tables (Table 1a,b). 
a)

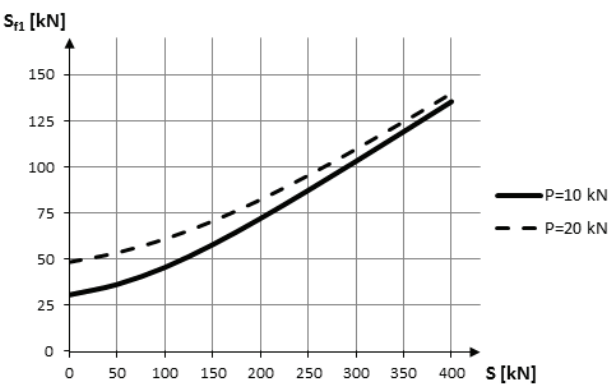

b)

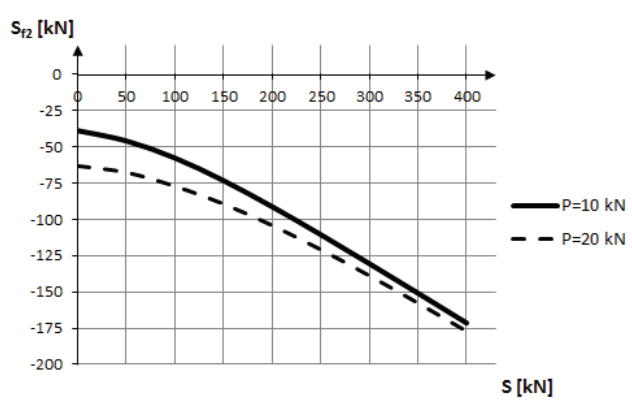

Fig. 5. Tensegrity plate: a) geometry; b) influence of self-stress on characteristic displacement.

Table 1.

Values of internal forces in the selected elements: a) $\mathrm{P}=10 \mathrm{kN}$; b) $\mathrm{P}=20 \mathrm{kN}$.

a)

\begin{tabular}{|c|c|c|}
\cline { 2 - 3 } \multicolumn{1}{c|}{} & \multicolumn{2}{c|}{$\mathbf{S}_{\mathrm{f}}[\mathbf{k N}]$} \\
\hline \multirow{2}{*}{$\mathbf{S}[\mathbf{k N}]$} & Element 1 & Element 2 \\
\hline 0 & 30,71 & $-38,80$ \\
\hline 50 & 36,35 & $-45,95$ \\
\hline 100 & 45,62 & $-57,69$ \\
\hline 150 & 57,94 & $-73,29$ \\
\hline 200 & 72,14 & $-91,27$ \\
\hline 250 & 87,37 & $-110,54$ \\
\hline 300 & 103,14 & $-130,50$ \\
\hline 350 & 119,22 & $-150,84$ \\
\hline 400 & 135,47 & $-171,41$ \\
\hline
\end{tabular}

b)

\begin{tabular}{|c|c|c|}
\cline { 2 - 3 } \multicolumn{1}{c|}{} & \multicolumn{2}{c|}{$\mathbf{S}_{\mathbf{f}}[\mathbf{k N}]$} \\
\hline \multirow{2}{*}{$\mathbf{S}[\mathbf{k N}]$} & Element 1 & Element 2 \\
\hline 0 & 48,48 & $-63,19$ \\
\hline 50 & 53,58 & $-67,71$ \\
\hline 100 & 60,97 & $-77,08$ \\
\hline 150 & 70,66 & $-89,37$ \\
\hline 200 & 82,32 & $-104,14$ \\
\hline 250 & 95,48 & $-120,81$ \\
\hline 300 & 109,68 & $-138,79$ \\
\hline 350 & 124,58 & $-157,66$ \\
\hline 400 & 139,95 & $-177,12$ \\
\hline
\end{tabular}

\section{DAMAGE DUE TO MEMBER LOSS AND SELF-REPAIR OF SMART TENSEGRITY STRUCTURES}

One of the properties of actively controlled structures is the capability of self-repair. The studies presented in this section prove that self-repair of structures like the tensegrity plate described above is possible using self-stress adjustment - it does not require any external interference.

All the performed analyses considered the plate presented in Figure 4a. Structural damage due to member loss was analysed. Removal of the cable marked in Figure 6 caused changes in the structure of modules 3 and 10, and as a result these modules could not be prestressed. Distribution of displacements before and after damage is presented in Figure $8 \mathrm{~b}$ and $8 \mathrm{c}$. The maximal displacement reached the value 55,29 mm, but 
the system was still able to carry loads. It can be observed that the cable removal did not cause collapse of the plate, as it was in case of a single module.

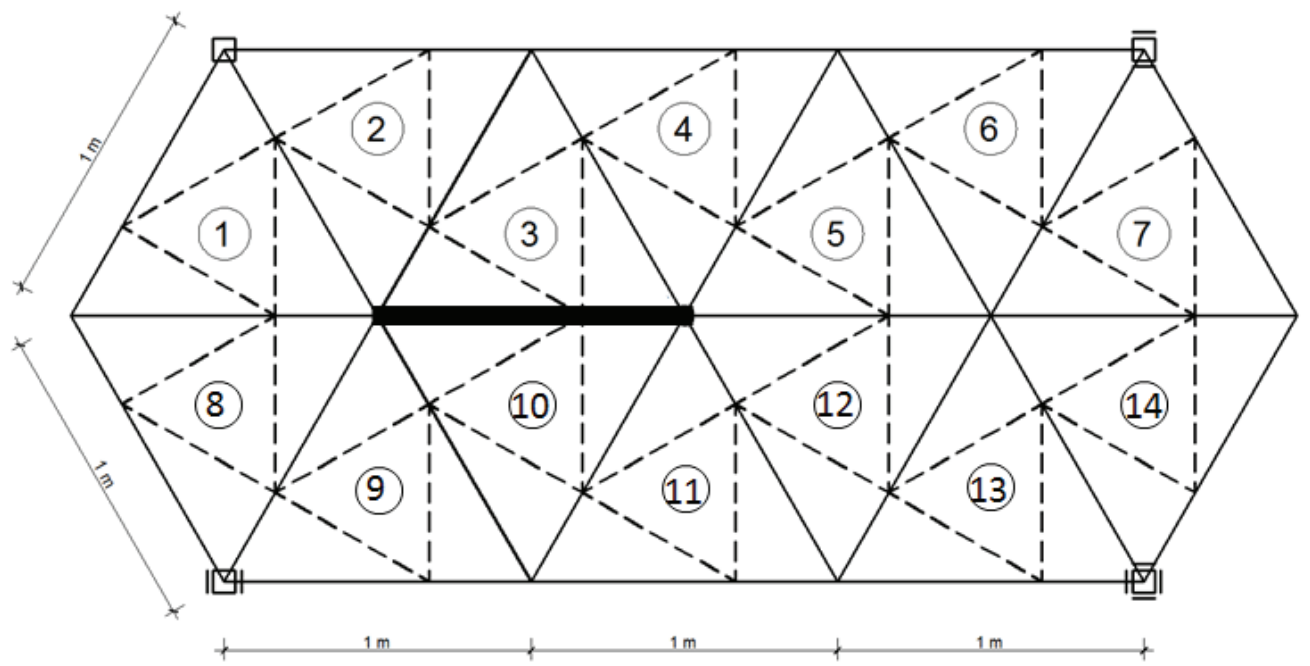

Fig. 6. Tensegrity plate with member loss (bold line).

One of the aims of this study was to prove that the damaged tensegrity structure can be repaired (return to the state from before damage) through an adjustment of self-stress in only one selected module. This adjustment was performed by applying additional prestressing forces in one of the modules. Figure 7 presents values of the prestressing forces in oblique cables of the particular modules, by which the repair understood as an elimination of an extra displacement occurs. The smallest force required to repair the plate was obtained for the self-stress adjustment of the module 11 (Figure 7).

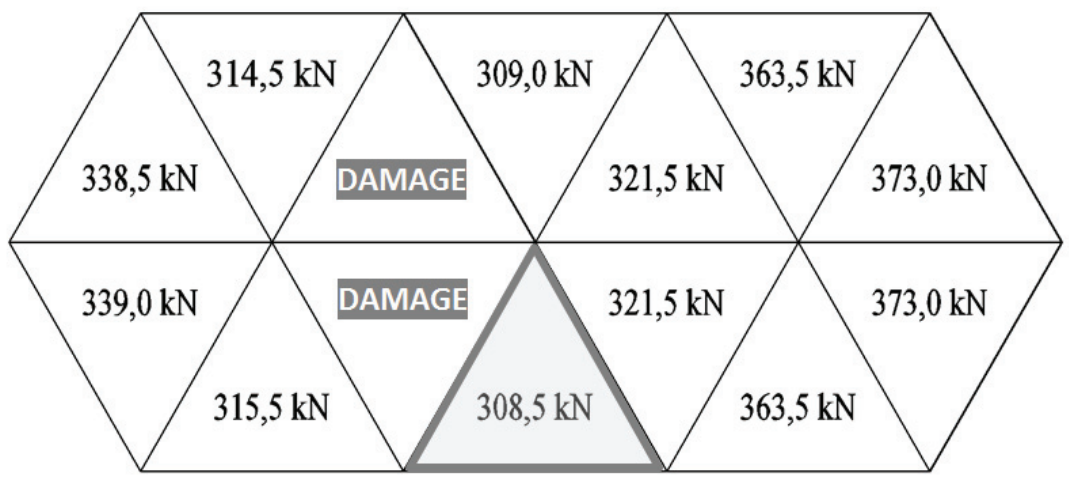

Fig. 7. Self-repair forces in oblique cables of the modules. 
The studies have proved that in case of the analysed plate, self-repair is possible without any external interference. Displacements of the structure return to the state from before damage after introducing additional prestressing forces into structural elements of the selected module. As far as the number of actuators needed in active control is concerned, it is important that the structural repair is feasible by controlling only one module, not the whole structure.

a)

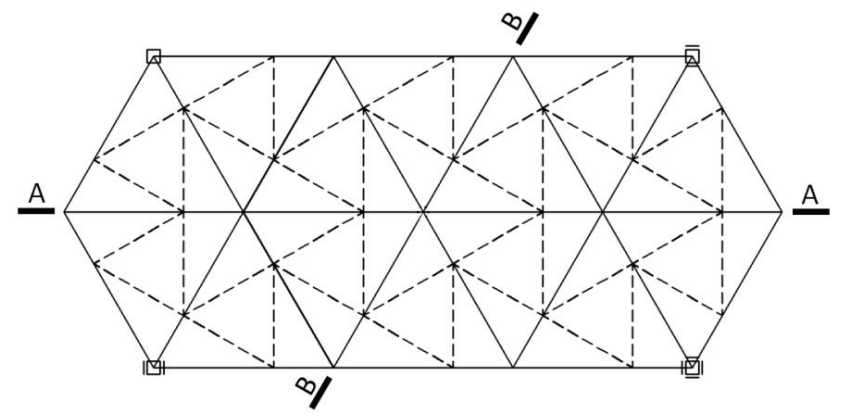

b)

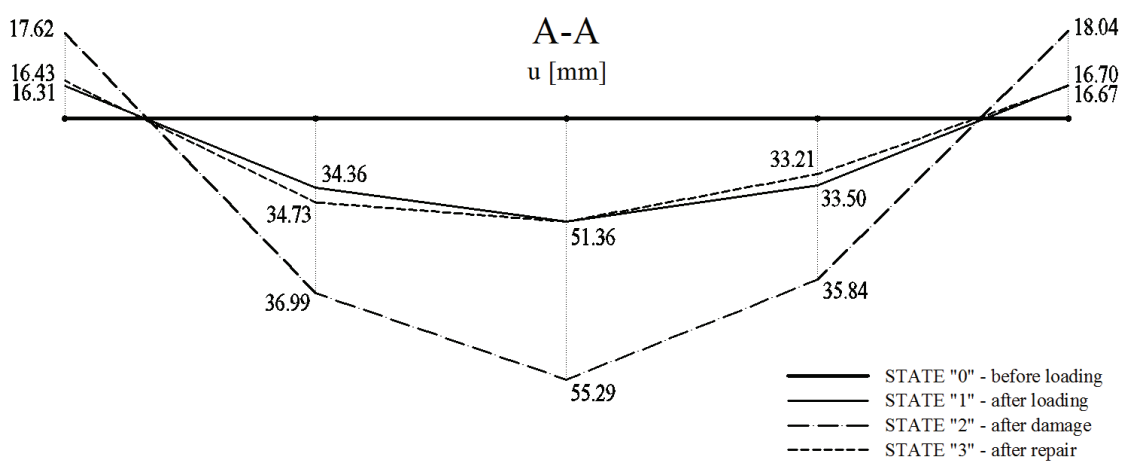

B-B

$\mathrm{u}[\mathrm{mm}]$

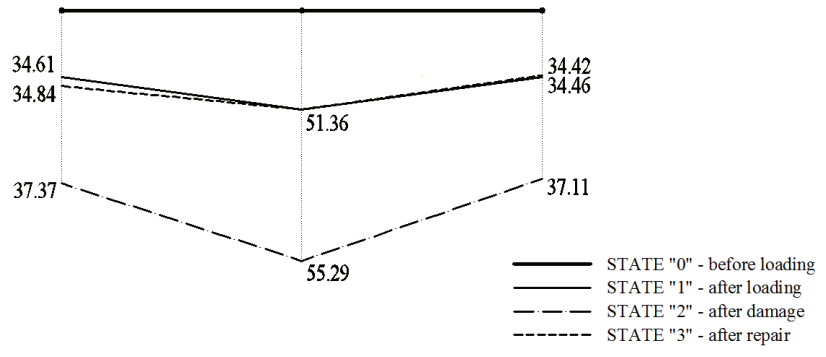

c)

Fig. 8. Distribution of displacements: a) cross sections; b), c) values. 


\section{Deployable tensegrity FoOtbridge AS AN EXAMPLE OF A SMART SYSTEM}

Smart systems have a wide range of applications in various areas - from aerospace engineering, through automotive industry, robotics and biomedical engineering, up to civil engineering. The last field of application is relatively new and so far, it is dominated by other areas. Nevertheless, there are a few fields within civil engineering, where smart systems are employed. One of them are deployable structures.

The structure presented below is a new type of a deployable bridge, based on tensegrity modules. A conceptual design of the footbridge was created paying special attention to the properties of tensegrity structures, which are prone to structural control using self-stress adjustment. Such structures may be used as temporary passages for pedestrians or single vehicles, they can have military applications and be employed in rescue services of different kinds and mountaineering.

The structure was built by analogy to the plate presented in Figure 4. It consists of reversed Simplex modules. The footbridge (Figure 9) has one simply supported span $8,00 \mathrm{~m}$ long, 2,77 $\mathrm{m}$ wide and $0,40 \mathrm{~m}$ high (structural height). The following data were used: strut lengths $0,81 \mathrm{~m}$, oblique cable lengths $0,48 \mathrm{~m}$, smaller triangle cable lengths $0,46 \mathrm{~m}$, bigger triangle cable lengths $0,80 \mathrm{~m}$. Struts were made of duralumin tubes with the following parameters: $\mathrm{Ea}=72 \mathrm{GPa}, \mathrm{Aa}=4,43 \mathrm{~cm} 2$, cables were made of steel with the parameters: $\mathrm{Es}=195 \mathrm{GPa}, \mathrm{As}=0,64 \mathrm{~cm}^{2}$.

The main aim of the presented footbridge is to provide passage for pedestrians over the given obstacle. Since the structure was designed to be used in special conditions (severe environment, rescue actions), the assumed pedestrian loading is $1,5 \mathrm{kN} / \mathrm{m}^{2}$, which is the value normally used for service passages.

The structure has one infinitesimal mechanism, which allows to control it by adjusting the values of prestressing forces. This special property of the designed footbridge enables extended applications of the structure, which apart from a normal pedestrian loading, can carry single vehicles. Such application of the footbridge is possible to achieve after proper prestressing of the active cables. The performed analyses proved that after applying self-stress on a certain level, the structure is able to carry vehicles such as: an off-road car Land Rover Defender $(1986 \mathrm{~kg})$ and a compact wheeled loader Bobcat S770 (4162 kg).

The values of prestressing forces introduced into structural elements are as follows: bigger triangle cables: $0,142134 \cdot \mathrm{S}$, smaller triangle cables: $0,246183 \cdot \mathrm{S}$, oblique cables: $0,256235 \cdot \mathrm{S}$, struts: $-0,256235 \cdot \mathrm{S}$, where $\mathrm{S}[\mathrm{kN}]$ is a multiplier of a prestressing force. When $S=10 \mathrm{kN}$ the structure is able to carry a single person. After applying $\mathrm{S}=100 \mathrm{kN}$, the assumed pedestrian loading as well as an off-road vehicle loading can be carried. To carry a compact wheeled loader, $\mathrm{S}=200 \mathrm{kN}$ has to be introduced.

This example shows perfectly the idea of an active structural control. The structure adjusts its properties by modifying the value of prestressing forces, thus allowing variable applications. The bigger the introduced forces are, the stiffer the bridge becomes and the more loading it can carry. 


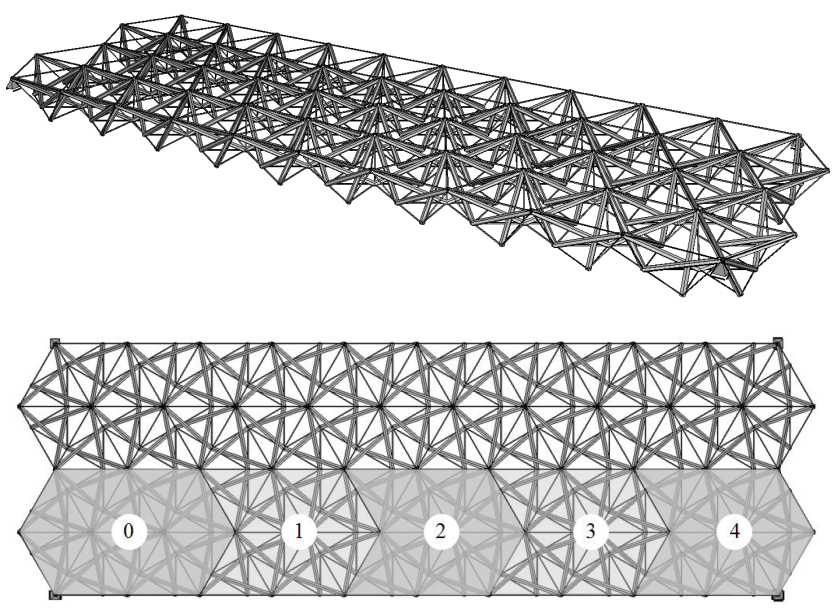

Fig. 9. Numerical model of the deployable tensegrity footbridge and its segmentation

Apart from an active control aspect, the footbridge was designed to be a lightweight deployable structure that is ready to be erected in the given location. In order to facilitate transport of the bridge, the whole structure was divided into assembly segments, each consisting of 10 (the first segment) or 8 (the following segments) Simplex modules (Figure 9). A scale model of one of such segments was made. The photographs (Figure 10) show how the structure should be erected.

a)

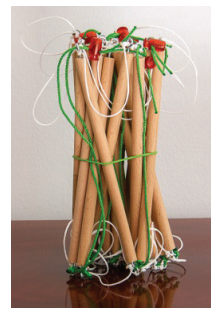

b)

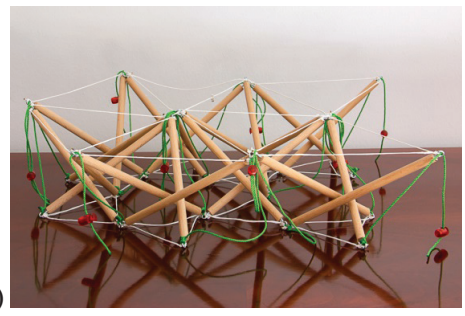

c)

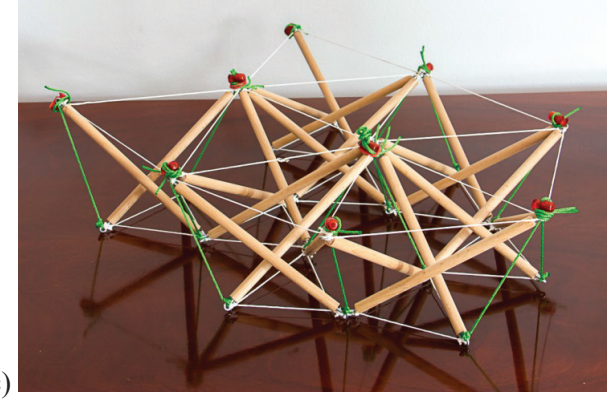

Fig. 10. Photographs of a scale model (1:5): a) folded segment; b) segment after erection before introduction of prestressing forces; c) segment after prestressing. 


\section{Conclusions}

The study discusses different aspects of an active structural control. It explains the idea of smart systems, introduces various terms used in smart technique and defines the structural smartness which, according to the author's opinion, should regard a structure itself, not the advanced technologies, with which buildings or bridges are equipped. Intelligent systems, understood in such way, may have various applications: vibration damping, reduction of displacements, acoustic isolation, damage localization and self-repair, stress reduction, etc.

Idea of the active structural control is presented on example of the smart tensegrity footbridge. The structure adjusts its properties by modifying the value of prestressing forces, thus allowing variable applications. The bigger the introduced forces are, the stiffer the bridge becomes and the more loading it can carry.

All the analyses presented in the study concern tensegrity structures, which are prone to the structural control through self-stress state adjustment. The paper introduces examples of structural control performed on tensegrity modules and plates. An influence of several self-stress states on displacements is analyzed and a study concerning damage due to member loss is presented. The studies have proved that self-repair of the analysed structures is possible without any external interference. Displacements of the structure return to the state from before damage after introducing additional prestressing forces into structural elements of the selected module.

Further research will be carried out on the application of actively controlled tensegrity structures in the field of civil engineering.

\section{REFERENCES}

1. W. Gilewski, A. Al Sabouni-Zawadzka, On possible applications of smart structures controlled by self-stress, Archives of Civil and Mechanical Engineering, 2014 - published on-line 16.09.2014.

2. G. Cazzulani, S. Cinquemani, L. Comolli, Enhancing Active Vibration Control Performances in a Smart Structure by Using Fiber Bragg Gratings Sensors, Proc. SPIE 8345, Sensors and Smart Structures Technologies for Civil, Mechanical and Aerospace Systems, San Diego, California 2012.

3. A.B. Strong, D.W. Jensen, Smart Structures - Impractical Or Inevitable? Presentation of Brigham Young University, 1999.

4. B. Adam, I.F.C. Smith, Learning, self-diagnosis and multi-objective control of an active tensegrity structure, Advances in Engineering Structures, Mechanics and Construction, Solid Mechanics and its Applications 140, 439-448, 2006.

5. S. Korkmaz, N. Bel Hadj Ali, I.F.C. Smith, Configuration Of Control System For Damage tolerance of a tensegrity bridge, Advanced Engineering Informatics 26, 1, 145-155, 2012.

6. L. Rhode-Barbarigos, N. Ben Hadj Ali, N. Motro, I.F.C. Smith, Designing tensegrity modules for pedestrian bridges, Engineering Structures 32, 4, 1158-1167, 2010.

7. C.M. Chang, B.F. Spencer, An Experimental Study of Active Base Isolation Control for Seismic Protection, Proc. SPIE 7647, Sensors and Smart Structures Technologies for Civil, Mechanical, and Aerospace Systems, San Diego, California 2010. 
8. G. Cazzulani, S. Cinquemani, L. Comolli, Enhancing Active Vibration Control Performances in a Smart Structure by Using Fiber Bragg Gratings Sensors, Proc. SPIE 8345, Sensors and Smart Structures Technologies for Civil, Mechanical, and Aerospace Systems, San Diego, California 2012.

9. K.C. Lu, J.H. Wenig, C.H. Loh, Turning the building into a smart structure: Integrating health monitoring, Proc. SPIE 7292, Sensors and Smart Structures Technologies for Civil, Mechanical, and Aerospace Systems, San Diego, California 2009.

10. R.E. Skelton, M.C. de Oliveira, Tensegrity Systems, London 2009.

11. R. Motro, Tensegrity: Structural Systems for the Future, Kogan Page Science, London 2003.

12. G.J.N. Juang, S. Sae-Ung, J.N. Yang, Active control of large building structures, Structural Control, H.M.E. Lipholz, ed., North-Holland, Amsterdam 1986.

13. A. Al Sabouni-Zawadzka, W. Gilewski, Control of Tensegrity Plate due to Member Loss, Proceedings of the XXIII Russian-Polish-Slovak Seminar: Theoretical Foundation of Civil Engineering, Wrocław 2014.

14. K. Wilde, Structural health monitoring systems supported by numerical models for civil engineering structures, Computer Methods in Mechanics, 17-18, Poznań 2013.

15. B.M. Phares, T.J. Wipf, U. Deza, J.P. Wacker, Development of a smart timber bridge - A five-year plan, USDA General Technical Report FPL-GTR-195, Madison 2011.

16. E.C. Eckhoff, V.M. Eller, S.E. Watkins, R.H. Hall, Interactive virtual laboratory for experience with a smart bridge test, Proc. of the 2002 American Society for Engineering Education Annual Conference \& Exposition, 2012.

17. G. Akhras, Nano \& Smart NDE Systems - Applications in Aerospace and Perspectives, $4^{\text {th }}$ International Symposium on NDT in Aerospace, 2012.

18. W. Gilewski, A. Kasprzak, Introduction to Tensegrity Modules Mechanics, in: Theoretical Foundations of Civil Engineering, Vol. I. Mechanics of Materials and Structures, 83-94, S. Jemioło, Sz. Lutomirski, eds., OW PW, Warsaw, 2012. 